\title{
Introduction to the detection technology of Ali CMB polarization telescope
}

\author{
He Gao ${ }^{1,2}$ - Congzhan Liu ${ }^{1}$ - Zhangwei $\mathrm{Li}^{1}$ - Yang Liu ${ }^{1,2}$ - Yongping $\mathrm{Li}^{1,2}$. \\ Siyu $\mathrm{Li}^{1,2}$ - Hong $\mathrm{Li}^{1}$. Guanhua Gao ${ }^{1,2}$. Fangjun $\mathrm{Lu}^{1}$ - Xinmin Zhang
}

Received: 6 April 2017 / Revised: 13 June 2017 / Accepted: 15 June 2017 / Published online: 3 July 2017

(C) The Author(s) 2017. This article is an open access publication

\begin{abstract}
Background Ali CMB polarization telescope (AliCPT) project is a well-timed and well-planned ground-based CMB project in Ali (Nagri) area of Tibet, China. It has been approved at the end of 2016.

Aims To give an introduction to the detection technology of AliCPT.

Method The whole receiver of AliCPT is introduced and discussed, including the optics, the cryostat, the preliminary design of focal plane TES bolometers, multiplexing SQUID readout, and so on.

Conclusions The raw sensitivity of $r$ will reach below 0.001 by 10 -year observation as AliCPT project being carried out and upgraded.
\end{abstract}

Keywords Microwave polarization detection -

Transition-edge sensor · Multiplexing SQUID readout

\section{Introduction}

\section{Primordial gravitational wave and CMB polarization}

LIGO Collaboration announced the first detection of the gravitational wave (GW) on February 11, 2016; it is the first time that GW was detected since general relativity (GR) was proposed by Albert Einstein 100 years ago. The discovery of GW has filled the final puzzle of GR prophecy, opening a

\section{Congzhan Liu}

liucz@ihep.ac.cn

1 Institute of High Energy Physics, Chinese Academy of Sciences, Beijing, China

2 University of Chinese Academy of Sciences, Beijing, China new era of $\mathrm{GW}$ research. A few experiments which aim to detect GWs from celestial motions at different frequencies are under development, for example, the projects of eLISA (evolved Laser Interferometer Space Antenna), Tian Qin, Tai Chi and so on. Different from the celestial sourced GWs, the primordial gravitational wave (PGW) which is the production from the quantum fluctuations of space and time at the most beginning of universe remains to be undiscovered yet. It is commonly believed that detection of PGW would open a new window into the physics of early universe. PGW corresponds to the most low frequency of $\mathrm{GW}$, since the wavelength is stretched into the size of the universe with the cosmic evolution from the most beginning to present. The efficient way for probing PGWs is to observe a special polarization pattern, the so-called B modes, in CMB.

In the standard model of cosmology, our universe experienced a period of accelerated expansion at the very beginning, and it provides a wonderful paradigm for understanding the origin of the primordial density perturbations which grew into the tiny $\mathrm{CMB}$ anisotropies as well as the large-scale structure around us at present. In addition to primordial density perturbations, the PGWs, corresponding to the primordial tensor perturbations, are generated at the same time. PGWs have been imprinted in the CMB B modes polarization pattern; thus, the detection of PGWs provides us an efficient way for exploring the early universe. The signal which is encoded in the tensor-to-scalar ratio parameter, $r$, is the main quantity for the direct measurement and reflect the amplitude of PGWs. The upper limit of the ' $r$ ' is $r<0.07$ at $2 \sigma$ C.L. $[1,2]$ given by the joint analysis of BICEP2 and Keck Array with Planck.

Ali CMB polarization telescope (AliCPT) aims to open the new observational window for PGWs with a ground-based CMB polarization telescope and measure the PGWs with high accuracy. The designed scientific goal of AliCPT is to 


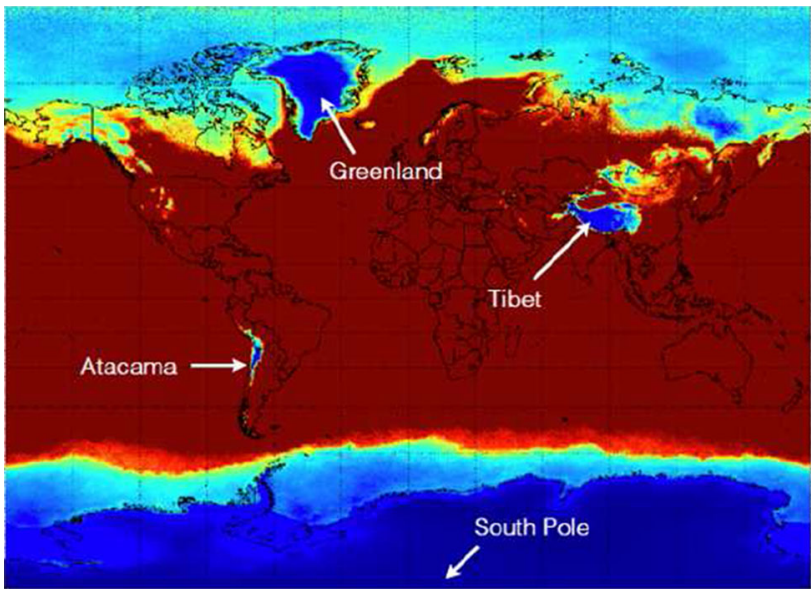

Fig. 1 Global water vapor distribution [4]

push the upper limit on tensor-to-scalar ratio to $\sigma(r)<0.001$ at $1 \sigma C . L$.

\section{AliCPT}

CMB observation asks for crucial dry environmental condition. Put experiments into space is a good choice; however, it costs lots of time, manpower and resources. The developing space CMB B-mode polarization satellite LiteBIRD perhaps produce results as early as the year 2025 [3]. The most important limitation for ground-based observation is the broadband absorption of the total water vapor in the atmosphere, often expressed as the precipitable water vapor (PWV). Even very tiny water content can lead to plenty of contamination to the $\mathrm{CMB}$ radiation and the reduction in atmospheric transmissivity. The selection of the observation band is strongly dependent on the PWV, especially for higher observation band.

Only four places on the earth are suitable for performing the ground-based CMB observations, which is shown in Fig. 1, and they are [4]: the South Pole and Atacama Desert in the Southern Hemisphere, and the Tibet and Greenland in the Northern Hemisphere. Tibet provides the best CMB observational site in North Hemisphere.

So far, almost all the CMB detection telescopes are sited in the Southern Hemisphere, such as the BICEP (Background Imaging of Cosmic Extragalactic Polarization) series in the South Pole and the ACT (Atacama Cosmology Telescope) in Chile. However, according to the result of the Planck satellite, there exist large low-foreground areas in the Northern Sky with much less foreground contamination compared to the Southern Sky. There is no CMB telescope in the Northern Hemisphere yet. It has become an emergent requirement in the CMB detection field to build telescopes in the Northern Hemisphere in order to achieve cooperative observation. Infrastructure of Greenland is incomplete; besides,

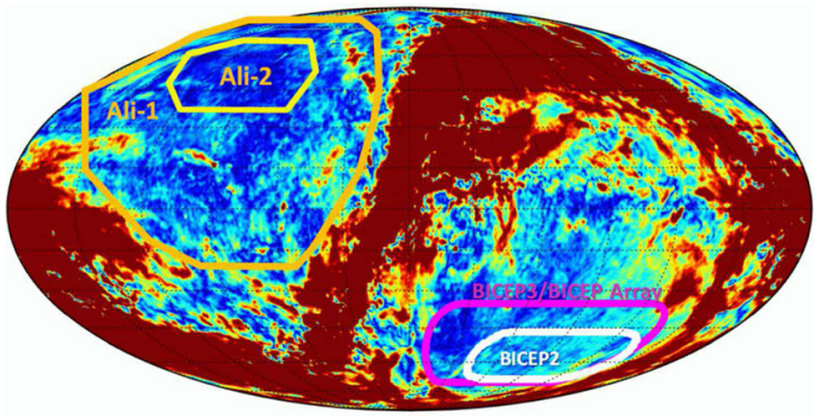

Fig. 2 Large low-foreground radiation areas in the Northern Hemisphere

Ali (阿里) (80.0 E, 32.3 N)・DJF Transmittance Quartiles

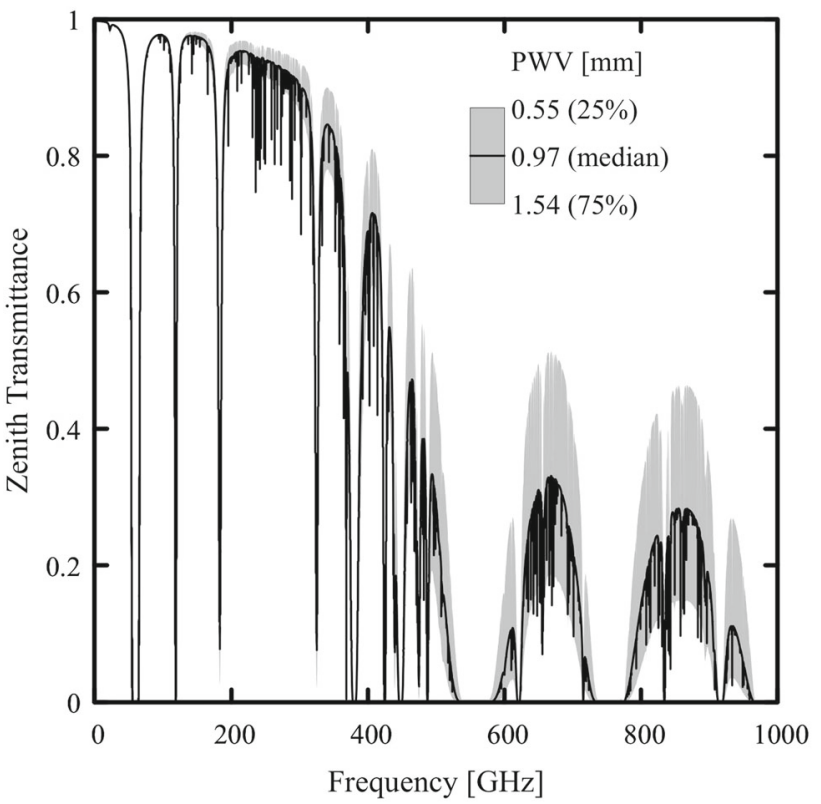

Fig. 3 Ali (winter) transmittance vs frequency band (from Scott Paine, Smithsonian astrophysical observatory)

supplies and transportation are big problems. In contrast, the Ali (Ngari) area of Tibet has very good transportation and infrastructure. These make Ali become the only suitable site for CMB observation in the Northern Hemisphere. Figure 2 shows large low-foreground areas in the Northern Sky, and Fig. 3 shows the Ali transmittance-frequency relationship in winter season, derived from reanalysis of the statistics data of MERRA (Modern-Era Retrospective analysis for Research and Applications).

Ali CMB polarization telescope (AliCPT) project is a ground-based CMB observation project led by Institute of High Energy Physics, Chinese Academy of Sciences (IHEP, CAS), cooperated with Stanford University, SLAC National Accelerator Lab. It has just been approved at the end of 2016 and enters into the developmental stage. 
AliCPT-1 is designed to observe the polarization of CMB at the two main central bands of 95 and $150 \mathrm{GHz}$.

The in-band microwave enters into a telescope and is focused on the focal plane. It is received by antenna and split by orthomode transducers (OMTs). One pair of split signals is transmitted to an isolated island by waveguide and transformed into heat by microstrips, next the heat is deposited at the ultra-sensitive TES (transition-edge sensor) detectors, and finally, the TES signals are readout by multiplexing SQUID (superconducting quantum interference device) amplifiers. In this way, we get the information of the input microwave power of two orthogonal orientations; the telescope will also rotate by $45^{\circ}$ during the observation to get another pair of signals so that the Stokes parameters such as $Q$ and $U$ can be extracted.

The E-mode and B-mode polarization information and angular power spectrum can be extracted by spherical harmonic expansion based on $Q$ and $U$. After many months data accumulation, multifrequency sky maps can be created, and therefore, foreground separation can be made. Eventually, CMB polarization sky map will be created and the range of ' $r$ ' will be acquired after a series of data processing.

AliCPT-1 will be located at an altitude of about $5250 \mathrm{~m}$. It will scan large areas of the sky, covering $60 \%$ of the Northern Sky in order to seek for very-low-foreground sky patches. AliCPT-2 will be at an altitude of about $6000 \mathrm{~m}$, where the PWV is even lower and the measuring accuracy will be improved to a quite higher level. It will make deep observations in small sky patches (as shown in Fig. 2).

Overall, Ali Project is a well-timed and well-planned project. In addition to hunt for PGWs, it can also provide independent tests for fundamental physics, including E-mode maps/science (isocurvature perturbations), and accurately study the reionization history; testing CPT symmetry by CMB polarization measurement; foreground study in the Northern Hemisphere.

\section{Receiver}

The core detectors on AliCPT's focal plane are TES bolometers. The price to make use of the ultra-sensitive detectors is that we have to establish a cryostat receiver, which contains microwave optical system, sub-Kelvin cryogenic system and focal plane detector modules.

\section{Optical design}

AliCPT's optical system consists of a microwave window, two lens, multilayer reflective infrared filters and so on. Its telescope will adopt a two-lens on-axis refracting type, which can be easily rotated. This is an excellent choice for polarization observation due to the need to get the $Q$ and

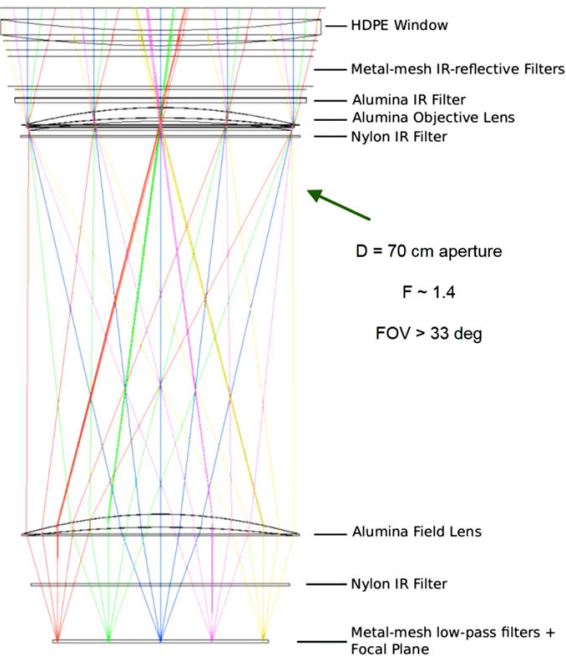

Fig. 4 Optical path of the microwave optical system

$U$ parameters, the reduction in the systematic error and the larger field of view compared to the reflecting type. Multilayer reflective infrared filters will be installed for reflecting the out-band radiation and reducing the radiation heat load in case of badly working of the cooling system. This design is more advantaged than the absorptive filters especially for bigger diameter and larger array of detectors. The optical design of AliCPT will inherit that of BICEP3 and further upgrade will be made. The most difficult parts for the microwave optical system might be the material selection and dicing of the anti-reflective coating for the lenses and filters. Figure 4 [2] shows the schematic of optical path.

The objective is designed to be an about 70-cm-diameter aperture. On the focal plane, 1712 TES bolometers are planned to be fabricated for each module in a 6-inch wafer which will be cut to a hexagonal shape, this restricts the pixel separation to be about $5.6 \mathrm{~mm}$. According to the so-called $2 F \lambda$ criterion $[5,6]$,

$2 F \lambda=\Delta x$

For $\lambda=2 \mathrm{~mm}(150 \mathrm{GHz})$, the criterion can be roughly achieved with $F=1.4$ ( $F$ is the focal ratio) and the focal length of about $980 \mathrm{~mm}$. It will be a faster system than BICEP3. The field of view will be more than $33^{\circ}$.

\section{Sub-Kelvin cryogenic system}

TES bolometers need the sub-Kelvin cryogenic system because they work at the transition region between the superconducting and normal state, usually in the order of a few hundred millikelvin. To reach a sub-Kelvin temperature, the first step is always to drop to the quasi- $4 \mathrm{~K}$, and the second step is to go down below $1 \mathrm{~K}$. 
Fig. 5 Feedhorn of ABS (left) [9] and AdvACT's OMT (right) [8]
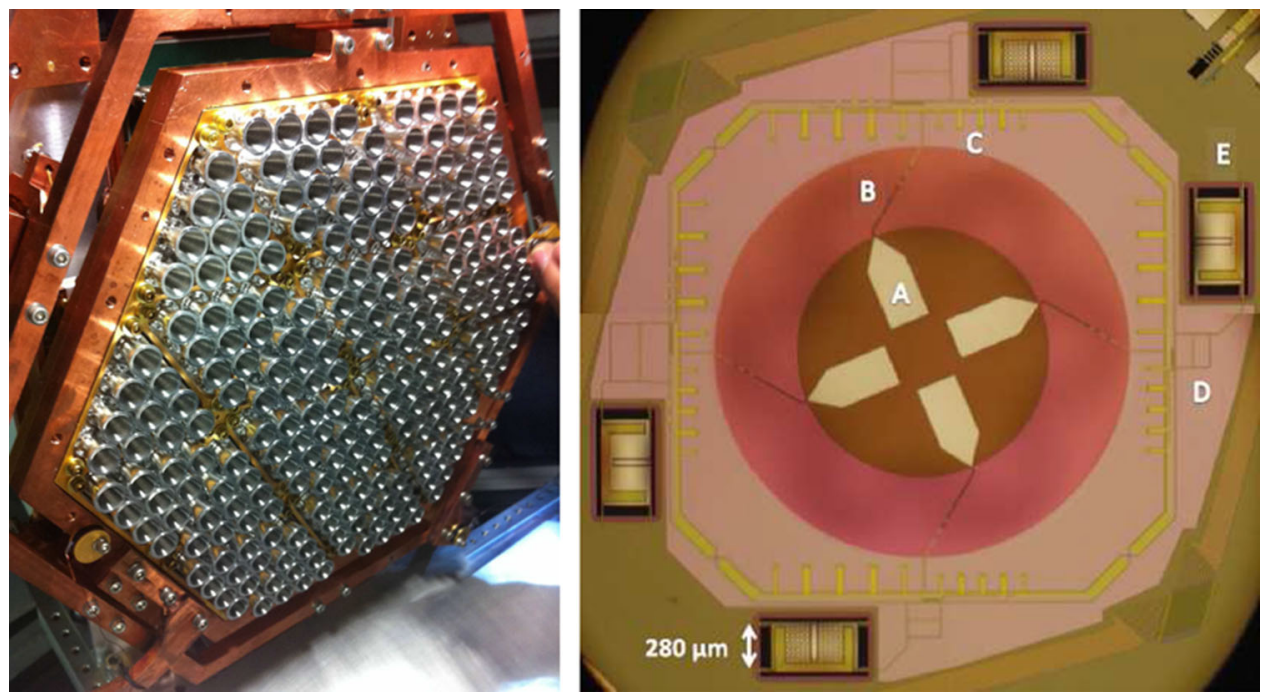

Step 1 AliCPT's 4K stage will adopt a commercial pulse tube refrigerator PT420 made by Cryomech Inc. The reason for this selection is to get a cooling power of more than $1.5 \mathrm{~W} @ 4.2 \mathrm{~K}$ at the $4 \mathrm{~K}$ stage.

Step 2 AliCPT will adopt a multistage sorption refrigerator, which utilizes the helium (He-4/He-3/He-3 3 stages) gas volatilization for cooling. The lowest temperature will be about $250 \mathrm{mK}$. Only the focal plane can go down below $1 \mathrm{~K}$. The TES working temperature is probably around $400 \mathrm{mK}$. It cannot work continuously due to the cycle of the release and absorption of the helium gas from and onto activated charcoal. There are many reasons for choosing the multistage sorption refrigerator rather than a dilution refrigerator, mainly because of the cost, the rotation of the receiver and no need to continuously work that last for many weeks. 3 days of one cooling cycle is enough to meet the requirement of a long period of observation.

\section{Focal plane detectors}

Focal plane detectors mainly consist of the OMTs, TES bolometers and SQUID amplifiers.

\section{Antenna and OMT}

To take the microwave measurement, antenna and waveguide are necessary. There are many types of antenna design, for example, BICEP2\&3 [7] used the planar slot antenna; BICEP1, AdvACT (Advanced ACTPol: the third generation of ACT) [8] and ABS (Atacama B-mode Search) [9] used feedhorn. AliCPT will adopt feedhorn. Feedhorn has more microwave coupling efficiency, the effect to reduce sidelobe is also very good and near gauss beam profile can come out. But it demands high machining precision.
To measure the polarization, OMT will be added below AliCPT's feedhorn in order to split received signals. Each OMT will be surrounded with 4 TES bolometers to detect the signals of two orthogonal orientations in two central bands of 95 and $150 \mathrm{GHz}$.

Figure 5 shows the feedhorn of ABS [9] and the OMT of AdvACT [8]. AliCPT will adopt a similar style. Simulations will provide very good guidance to the design of antenna and OMT.

\section{TES}

AliCPT's focal plane TES (transition-edge sensor) detector is one of the most sensitive detectors which have been studied for more than 20 years around the world. The essence of TES is a kind of thermal equilibrium detectors that are extremely sensitive to the change in the temperature. The detection range of TES goes from low frequency $\mathrm{THz}$ all the way to gamma ray. Especially in the microwave and infrared band, it has been widely used as TES bolometers. In the field of X-ray detection, spectrometers based on TES microcalorimeters have achieved an energy resolution level of about $2 \mathrm{eV} @ 6 \mathrm{keV}$ [10], and nearly no degraded performance with the increase in photon energy. TES microcalorimeters need specialized absorbers for the energy deposition of the X-ray or gamma ray.

Figure 6 (top) shows the schematic of a thermal equilibrium detector (top left) [11], the ideal $R-T$ curve (top middle) and the voltage-biased circuit connection of a TES (top right). The TES initially stays at a point where the resistance is usually between $0.1 R_{n}$ and $0.8 R_{n}\left(R_{n}\right.$ : the normal resistance). The TES branch is voltage-biased due to the condition of $R_{\text {bias }} \ll R_{\text {TES }}$, and therefore, nearly all the current $I_{\text {bias }}$ goes through the $R_{\text {bias }}$, and the $V$ (voltage across the TES branch) keeps constant. When some power input or energy deposited 
Fig. 6 Schematics of a thermal equilibrium detector (top left) [11]; $R-T$ curve of an ideal TES (top middle); voltage-biased TES circuit (top right); $T-t$ curves of a typical simple calorimeter (bottom left) and a bolometer (bottom right)
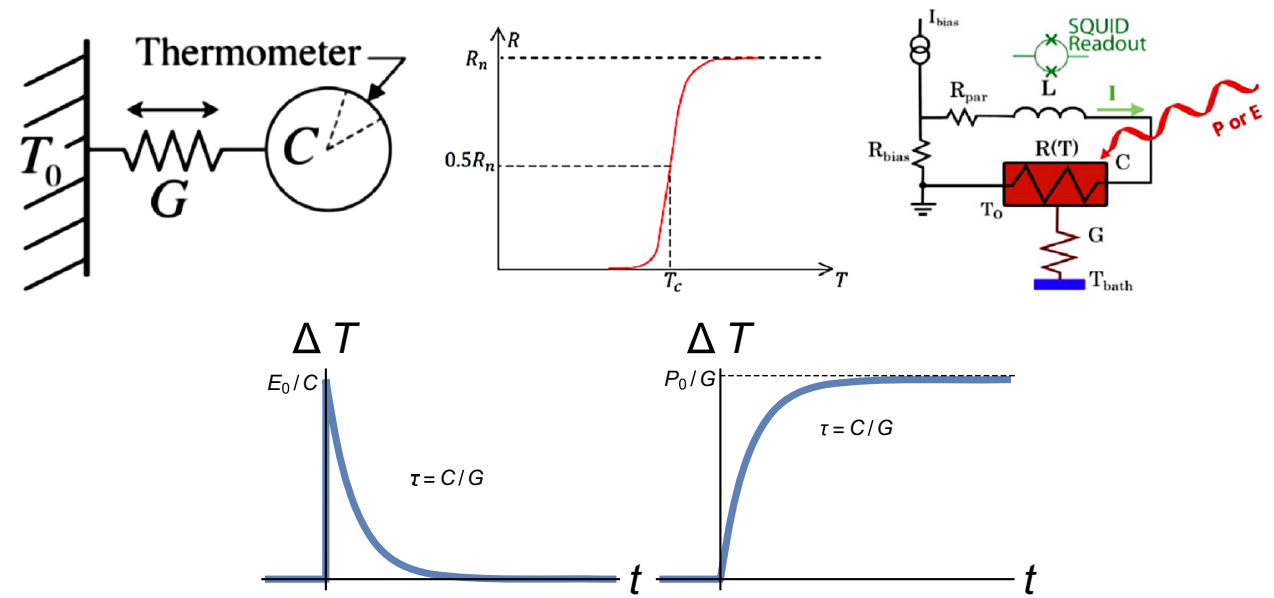

on the absorber, the TES gets heated, so the temperature increases. The intrinsic time constant is $\tau=\frac{C}{G}$, where $C$ and $G$ are the heat capacity and the thermal conductance of the device. The bath temperature is $T_{b}$; we can use a simple thermal differential equation to describe this increase in temperature:

$C \frac{\mathrm{d} T}{\mathrm{~d} t}=-G\left(T-T_{b}\right)+P_{\text {in }}$

where the Joule heat of the TES is ignored and the whole process of thermal conduction is simplified. Depending on the form of the input power $P_{\text {in }}$, the solutions are different.

If the $P_{\text {in }}$ is a impulse function, the temperature change is:

$\Delta T=\frac{E_{0}}{C} e^{-\frac{t}{\tau}} u(t),\left(P_{\text {in }}=E_{0} \delta t\right)$

Here, $u(t)$ is the unit step function.

If the $P_{\text {in }}$ is a step function, the temperature change is:

$\Delta T=\frac{P_{0}}{G}\left(1-e^{-\frac{t}{\tau}}\right) u(t),\left(P_{\text {in }}=P_{0} u(t)\right)$

The input is usually the former pulse form when detecting a single photon such as a X-ray photon, and this kind of device is called the calorimeter. However, the power input is usually the latter form corresponding to a bolometer. Figure 6 (bottom) shows the two responding temperature curves. For AliCPT, the TES detects the power of input microwave, so the latter equation can simply describe the process of temperature increase.

Imagine that a microwave signal enters into a TES's island and is transformed into heat by the meandering microstrips; therefore, the temperature of the TES bolometer nearby changes, and the TES's resistance changes a lot due to the sharp $R-T$ curve [Fig. 6 (top middle)]; therefore, the current goes through the TES branch changes and this faint change can be measured accurately by the SQUID amplifier.

For practical TES circuit, it will form a strong negative electrothermal feedback which leads to increased stability, linearity, dynamic range, time response and so on. The general equations and solutions are described in [12], and the response of the current of a single TES to the input power $\mathrm{P}$ can be given in the limit of small signal analysis [7,12]:

$s_{I}=\frac{\delta I}{\delta P}=-\frac{1}{V}$

To conclude, the TES's current has a very linear response to the input microwave power, and the SQUID amplifier has a linear response to the input current of the TES too. In this way, we get the accurate magnitude of the input power.

TES's performance is also relevant to $T_{c}$; often, $T_{c}$ is expected to be a relatively lower value to reduce the noise. AliCPT's TES material is probably about 400 millikelvin.

One single TES film's size is usually in the order of a hundred microns, and the thickness is in the order of a hundred nanometers. Such size of TES films with OMTs is made by micronanofabrication in wafers, so are the SQUID devices.

There are often 3 choices [12] for the material selection of TES: elemental superconductors, bilayers or multilayers and alloy superconductors. In the CMB observation field, all of the three types are in use, such as Ti TES in BICEP2\&3, $\mathrm{Mo} / \mathrm{Cu}$ bilayer in ACTPol, Al-Mn Alloy in AdvACT.

AliCPT will adopt the Al-Mn Alloy as its TES material. The Al-Mn alloy's $T_{c}$ can vary from below $100 \mathrm{mK}$ to a few hundred millikelvin depending on the mixture ratio, the thickness and the bake temperature during a process [8] of sputtering. Dropping the $T_{c}$ to some extent will improve the noise performance of the TES. Moreover, the Al-Mn TES shows better uniformity and very small influence with different magnetic field.

TES is usually microfabricated on a $\mathrm{SiN}$ wafer, and the structure is formed after a series process of film deposition, 


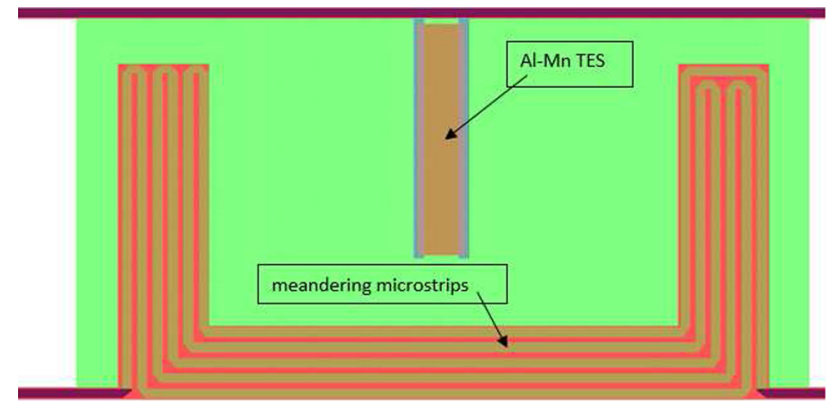

Fig. 7 Schematic of an Al-Mn TES island; signals are transformed into heat by meandering microstrips, and the current of TES changes with the changes in the temperature and resistance of TES

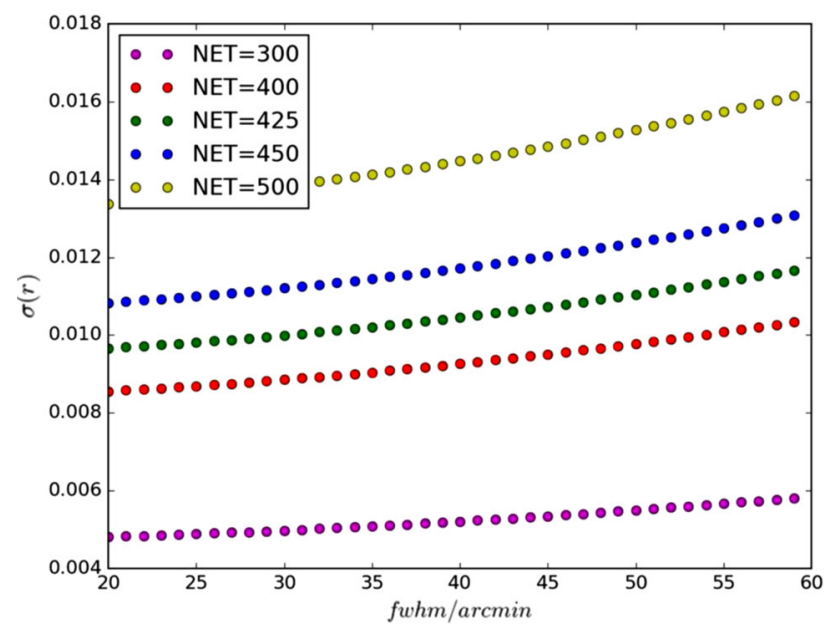

Fig. 8 Simulated relationship between the $\sigma(r)$ and beam width at different NET $\left(\mu \mathrm{K}_{\mathrm{CMB}} \sqrt{\mathrm{s}}\right)$ values

lithography, etching, liftoff and so on. The process of film deposition and lithography defines the size and thickness of TES, which determines the $R_{n}$ and $T_{c}$.

The most difficult parts may be the etching of $\mathrm{SiN}$ and the DRIE (deep reactive ion etching) of silicon to form the TES island. Figure 7 shows a schematic picture of an Al-Mn TES island (similar to the TES used in AdvACT [8]); in the middle is the Al-Mn alloy film, the whole $\mathrm{SiN}$ is etched, and only the island and four legs are left. The purpose of the etching of the $\mathrm{SiN}$ is to decrease the thermal conductance in order to make the response slower due to not very fast signal of microwave power input compared to single-photon energy input.

For AliCPT-1, at least 4 modules are planned to be installed at first, each with 1712 detectors; therefore, $N$ is at least 6848 in the beginning. The short-term goal of AliCPT1 is to constrain the $\sigma(r)$ to below 0.01 within 3 years, and this goal relays on the noise equivalent temperature (NET) and the beam width. Figure 8 shows the simulated relationship between $\sigma(r)$ and the FWHM beam width at different $\mathrm{NET}_{\text {per-detector values, where the observation efficiency }}$ is $10 \%$, the covering sky is $60 \%$, the number of detector counts is 6848 and the observation time is 3 years. When $\mathrm{NET}_{\text {per-detector }}$ is less than $400 \mu \mathrm{K}_{\mathrm{CMB}} \sqrt{\mathrm{s}}$, the short-term goal can be achieved no matter what the beam width is. So we set the goal of $400 \mu \mathrm{K}_{\mathrm{CMB}} \sqrt{\mathrm{s}}$ for each device. The combined NET is roughly reduced by a factor of $\sqrt{N}$ to be less than $5 \mu \mathrm{K}_{\mathrm{CMB}} \sqrt{\mathrm{s}}$, where $N$ is the number of detector counts.

The NET derivation from time-ordered data (TOD) is described in [13], usually the NET scales with the noise equivalent power (NEP) at a certain central band [14], gives a simple general relationship between the $\mathrm{NET}_{\mathrm{CMB}}$ and the NEP:

$$
\mathrm{NET}_{\mathrm{CMB}}=\frac{\mathrm{NEP}}{\sqrt{2} \int d \nu \frac{\mathrm{d} B\left(\nu, T_{\mathrm{CMB}}\right)}{{ }^{\mathrm{d} T} \mathrm{CMB}} \frac{c^{2}}{\nu^{2}} \tau(\nu)(1-\epsilon(\nu))}
$$

where $B(v, T)$ is the Plank spectral brightness, $\tau(v)$ is the optical transfer function and $\epsilon(\nu)$ is the atmospheric opacity. It seems that it highly depends on the shape of $\tau(v)$, which is not determined before the telescope being made.

The NEP can only be roughly estimated to be at most $75 \mathrm{aW} / \sqrt{\mathrm{Hz}}$ for each single TES detector (refers to [15]). Here, we make a specific analysis of the total noise of the TES to give a preliminary design reference.

The main noise sources we considered for one detector are the photon noise, the phonon noise, TES Johnson noise, amplifier noise and excess noise. The $\mathrm{NEP}_{\text {total }}^{2}$ equals to the sum of $\mathrm{NEP}^{2}$ of each noise contribution.

The photon noise which is linked to the PWV is the dominant one. the photon noise equivalent power ( $\left.\mathrm{NEP}_{\text {photon }}\right)$ can be expressed [16] as a sum of Bose and shot noise contributions:

$\mathrm{NEP}_{\text {photon }}^{2}=2 h v P_{\text {load }}+\frac{2 P_{\text {load }}^{2}}{\Delta v}$

where $v$ is the band center, $\Delta v$ is the bandwidth and $P_{\text {load }}$ is the optical loading which equals to:

$$
P_{\text {load }}=2 \eta k_{B} T_{R J} \Delta v+P_{\text {internal }}
$$

$\eta$ is the optical efficiency. $k_{B}$ is the Boltzmann constant. The Rayleigh-Jeans brightness temperature $T_{\mathrm{RJ}}$ depends on the PWV and observation band, for about $148 \mathrm{GHz}$ and an elevation of about $50.5^{\circ}$ [13], $T_{\mathrm{RJ}}=(6.0 P W V+3.5) \mathrm{K}$. According to Ali area's condition, the average of the PWV in observation season is estimated to be no more than $1 \mathrm{~mm}$; here, we take the value of $1 \mathrm{~mm}$. As for the internal power, we will try to make it to a value of below $3 \mathrm{pW}$ to reduce the $P_{\text {load }}$, the optical efficiency is about $40 \%$ and the bandwidth is often selected to be about $37.5 \mathrm{GHz}$. Based on these, we get a $P_{\text {load }}$ of about $6.9 \mathrm{pW}$ and a NEP photon of about 
$62.8 \mathrm{aW} / \sqrt{\mathrm{Hz}}$ by calculation. For better observation, what we can do is to further reduce the $P_{\text {internal }}$ and choose the period of time when the PWV is very low.

The phonon noise is given by

$\mathrm{NEP}_{\text {phonon }}^{2}=4 k_{B} T_{c}^{2} G_{c} F\left(T_{c}, T_{b}\right)$

where the Mather factor $F\left(T_{c}, T_{b}\right)$ is roughly 0.5 [16].

To reduce the phonon noise, we can decrease the $G_{c}$ by controlling the etching of $\mathrm{SiN}$ or decrease the $T_{c}$ by controlling the film deposition. However, the saturation power must be taken into consideration in case that TES enters a nonlinear region; it is a trade-off. The saturation power is:

$P_{\text {Sat }}=\int_{T_{b}}^{T_{c}} G d T=G_{c} T_{c} \frac{1-\left(\frac{T_{b}}{T_{c}}\right)^{\beta+1}}{\beta+1}$

where $\beta$ is material dependent, usually around 2.1 [16].

The saturation power should be at least twice the $P_{\text {load }}$, that is, to be more than $13.9 \mathrm{pW}$. For AliCPT, the bath temperature is about $250 \mathrm{mK}$, and the $T_{c}$ is selected to be about $394 \mathrm{mK}$ to reach the lowest noise based on the relationships above. A $G_{c}$ of about $144 \mathrm{pW} / \mathrm{K}$ is needed (by controlling the etching of $\mathrm{SiN}$ and DRIE) to reach the target saturation power; then, we get a $\mathrm{NEP}_{\text {phonon }}$ of $24.9 \mathrm{aW} / \sqrt{\mathrm{Hz}}$. The exact value of $T_{c}$ and $G_{c}$ should be optimized after testing over and over again.

TES Johnson noise mainly depends on the $R-T$ transition curve, $T_{c}$ and the working point, and the $R-T$ curve usually depends on the quality of the TES film. The final noise performance of TES will be optimized after trying plenty of times in controlling the process of film deposition, for example, controlling the pressure, the source power or something. The SQUID noise is usually less than $3 \mathrm{aW} / \sqrt{\mathrm{Hz}}$, and the excess noise is not important (here the aliased noise is not considered). Under this circumstance, to achieve a total sensitivity as low as possible, the TES Johnson noise should be reduced by at least one order of the sum of the noise above, which is less than 1 or $10^{-18} \mathrm{~W} / \sqrt{\mathrm{Hz}}$. And the $\mathrm{NEP}_{\text {total }}$ is expected to be of $67.7 \mathrm{aW} / \sqrt{\mathrm{Hz}}$. It meets the requirement of less than $75 \mathrm{aW} / \sqrt{\mathrm{Hz}}$, and $\mathrm{NET}_{\text {per-detector }}$ is estimated to be about $350 \mu \mathrm{K}_{\mathrm{CMB}} \sqrt{\mathrm{s}}$.

Although the phonon noise and TES Johnson noise can still be optimized, the dominant noise is still the photon noise highly depending on the PWV. The best observation time comes when the local PWV is very low. Figure 9 shows the calculated relationship between the optimized $\mathrm{NEP}_{\text {total }}$ and the PWV based on the all the conditions above.

It is planned to establish a telescope array for AliCPT at the end, and each will be equipped with more than 20000 TES bolometers. The combined NET will be at a very high level of less than $3 \mu \mathrm{K}_{\mathrm{CMB}} \sqrt{\mathrm{s}}$, and only 1-year observation

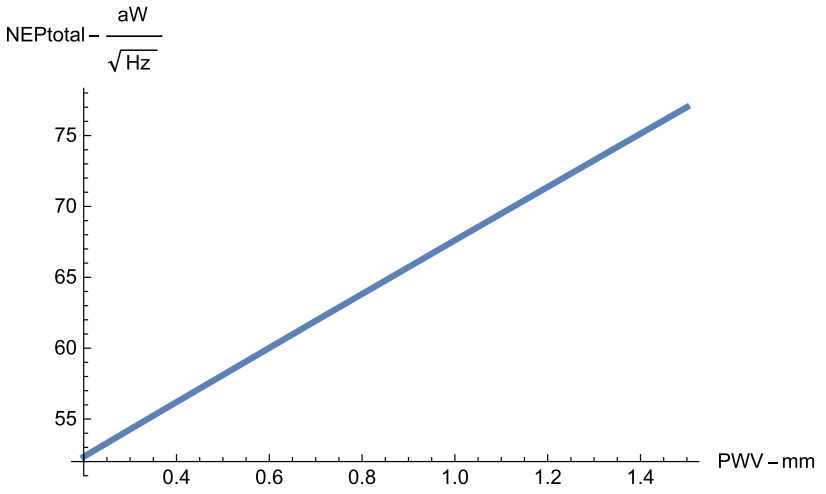

Fig. 9 Calculated relationship between the $\mathrm{NEP}_{\text {total }}(\mathrm{aW} / \sqrt{\mathrm{Hz}})$ and PWV (mm)

data will be comparable to that produced by small number of detectors in many years.

\section{SQUID readout}

A low input impedance amplifier is necessary to match the TES because of TES's very low resistance. Although TES comes out in 1940s, not until the creation of the SQUID amplifier, TES was widely used. The SQUID amplifier is almost the only choice for TES readout so far. For large array, each TES is equipped with at least one good SQUID.

SQUID (superconducting quantum interference device) is a type of sensors that is extremely sensitive to the magnetic field, it can be used to detect faint magnetic field or faint signals that can be transformed into magnetic flux. A SQUID amplifier consists of two parts. The first part is mainly the SQUID chip in the low temperature. The second part is a room-temperature electronics system.

dc-SQUID belongs to a kind of superconducting quantum devices. Figure 10 shows the schematic of a dc-SQUID and a piece of mask of a simple washer-type SQUID, and it consists of two parallel Nb-based Low- $T_{c}$ Josephson junctions with a closed loop and an annular holes.

If the SQUID is current-biased, the voltage across the SQUID will present a periodical sinusoidal-like relationship with the applied flux. Figure 11 shows the curve.

Almost every dc-SQUID readout is based on the fluxlocked loop (FLL) [17], and the dc-SQUID will be locked in a very small range around the working point $\mathrm{W}$ when external feedback electronics are connected to the feedback coil. Around the point $\mathrm{W}$, the output $\delta V$ will be proportional to the input $\delta \Phi_{a}$, if this SQUID amplifier is directly connected to the TES circuit with an input coil. The output voltage is:

$V_{\text {out }}=\frac{M_{\text {in }} I_{\text {in }}}{M_{f}} R_{f}$ 

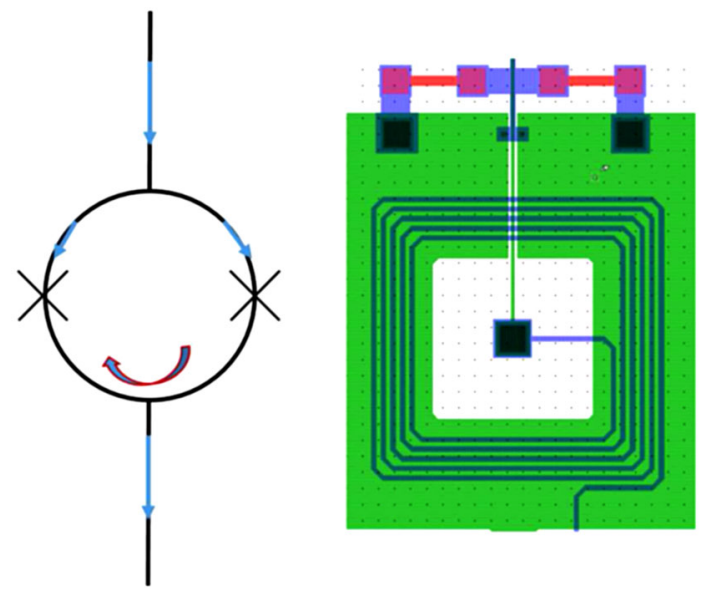

Fig. 10 Schematic of a dc-SQUID and the simple washer structure in a mask

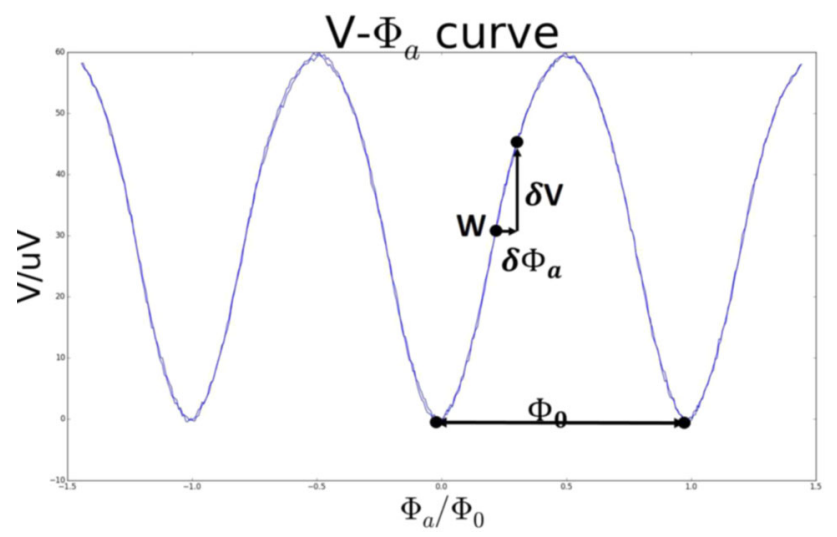

Fig. $11 \mathrm{~V}-\phi_{a}$ curve of a dc-SQUID fabricated in Tsinghua University; $\phi_{a}$ is input to the circuit by applying a current through the input coil (Fig. 10)

where $M_{\mathrm{in}}$ is the mutual inductance between the input coil and SQUID, $M_{f}$ is that between the feedback coil and SQUID, and $R_{f}$ is the resistance of the feedback resistor.

The SQUID exhibits a very linear trans-impedance amplification response to the input current $I_{\text {in }}$ and has nothing to do with the gain of the room-temperature electronics.

Commercial SQUID amplifiers [18] often take the twostage SQUID amplifier readout method for the readout of one or a few low-temperature detectors. However, for large array of TES detectors, multiplexing SQUID readout must be taken into consideration. Several multiplexing readout schemes for TES have been developed, including time-division multiplexing (TDM) [19], frequency-division multiplexing (FDM), code-division multiplexing (CDM) and the developing microwave SQUID multiplexing readout [20].

According to the AliCPT-1's situation, the most mature readout scheme TDM will be used.

Figure 12 [12] shows the TDM readout style, and each TES is voltage-biased, and connects a SQUID switch in

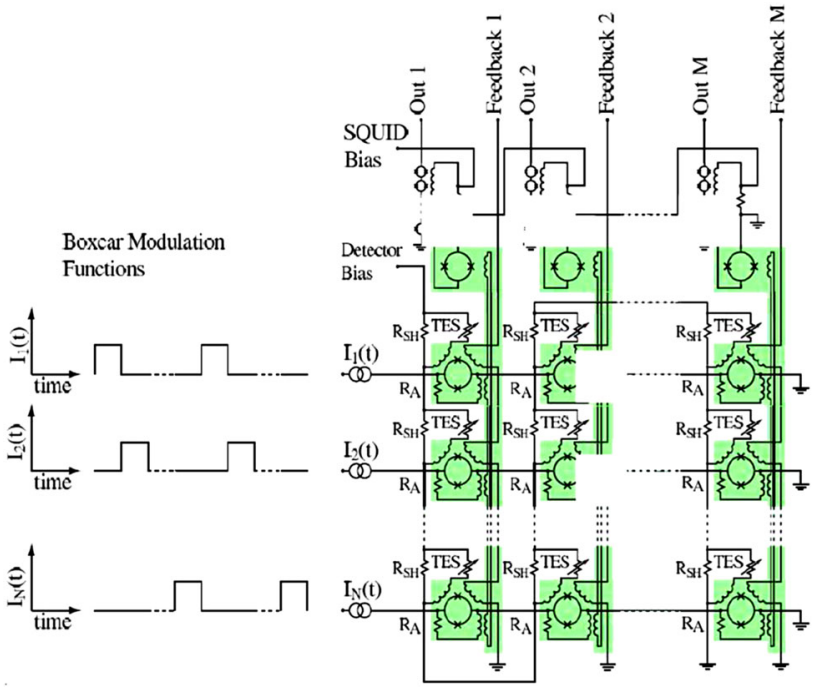

Fig. 12 Schematic of the time-division multiplexing SQUID readout [12]

the TES branch. Boxcar functions turn on a row of SQUID switches at one time, and each column output is inductively transformed into an output SQUID, and next transformed into the series SQUID array to match the room-temperature digital electronics. Finally the electronics gives feedback to this column of input SQUID to achieve the FLL mode. Although many new designs have been made, for example, BICEP3 adopted a new flux-activated switch [21], the style of TDM stays the same. The whole bandwidth of the systems is from tens of $\mathrm{MHz}$ to hundreds of $\mathrm{MHz}$. TDM factor will be at most 64:1. The detector counts of AliCPT- 1 are about 6848 at first, and TDM can still work well so that the observation data come out within 5 years can be guaranteed.

\section{Modularization}

SQUIDs are so sensitive to magnetic field that they always need the magnetic shielding. The OMT, TES and SQUID chip all need PCBs, shieldings, detector frame and the heat sink. All of these things will be integrated and packaged to make modularized detector structure in AliCPT. It is planned to make 4 detector modules for AliCPT- 1 at first; then, more modules will be added gradually, each of which contains 1712 detectors.

Figure 13 shows the hexagonal architecture detector modules, and this beehive-like structure makes detector tiles more compact and the electrical connection more ordered. It is very convenient for upgrade and repair. Without doubt, the packaging and modularization will be of difficulty.

The external auxiliary systems out of the receiver are mainly the temperature and electrical control system, data acquisition system, telescope base, etc. The electrical feed- 


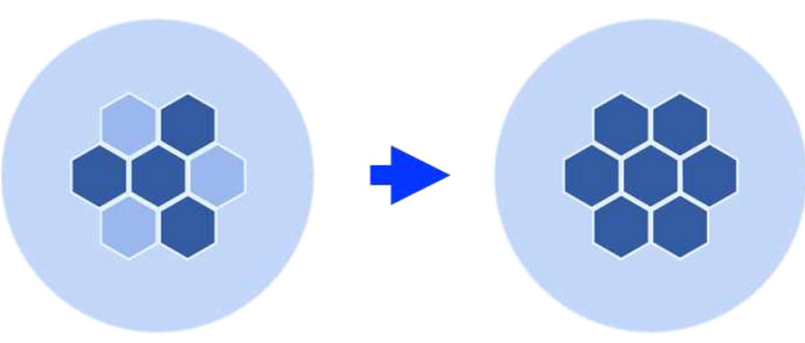

Fig. 13 Schematic of the hexagonal architecture detector modules

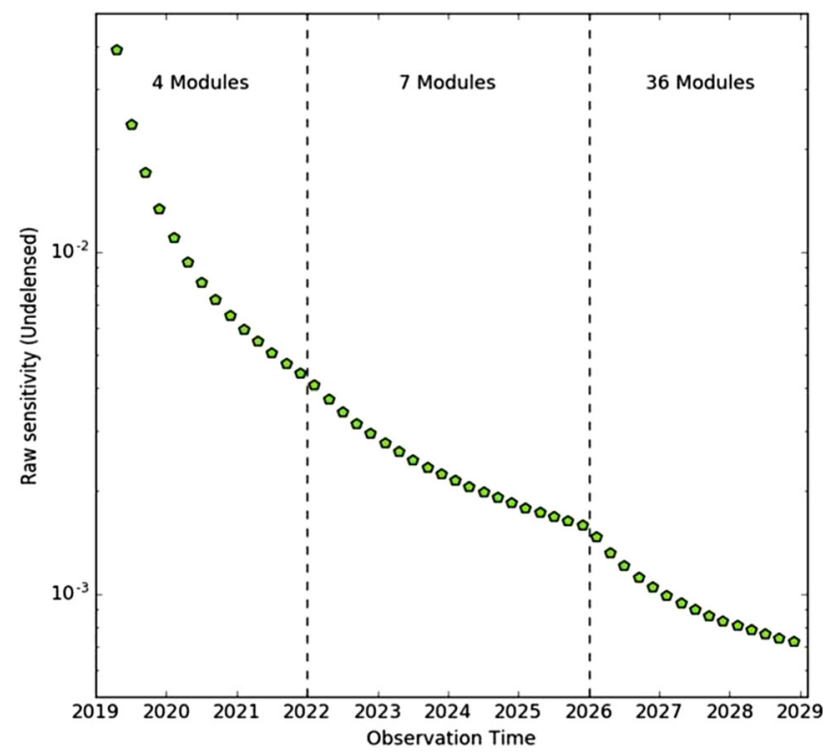

Fig. 14 Simulated relationship between $r$ raw sensitivity and the observation time through connections from inside to the external electronics are another important thing.

\section{Summary and prospect}

The final number of modules in AliCPT-1 (Advanced AliCPT-1) will be at least 7; at that time, the number of detector counts will be more than 11000 .

For AliCPT-2, the plan is to establish the AliCPT array at the end of the project, each containing at least 12 modules (probably 19). After AliCPT-2 installed, the detection precision will be at a very high level and the $\sigma(r)$ be further improved. Besides, the new layout of microwave multiplexing (based on RF-SQUID) scheme will be adopted for the readout of the very large number of detectors, its bandwidth will reach more than a few $\mathrm{GHz}$ and the multiplexing factor may be over 1000:1. Figure 15 shows the timeline of the AliCPT Project. By about 10-year observation, the raw sensitivity of $r$ will reach below 0.001 . Figure 14 is the simulated relationship between the r raw sensitivity and the observation time based on the $350 \mu \mathrm{K}_{\mathrm{CMB}} \sqrt{\mathrm{s}}$ of each detector's NET. Figure 15 shows the timeline of the AliCPT project.

This paper gives a brief introduction to the technology and instruments of AliCPT. AliCPT will play a lead role in the CMB detection field in China. There is a big probability that AliCPT will detect the PGW and make great breakthroughs in many relevant scientific fields.

Fig. 15 AliCPT's timeline

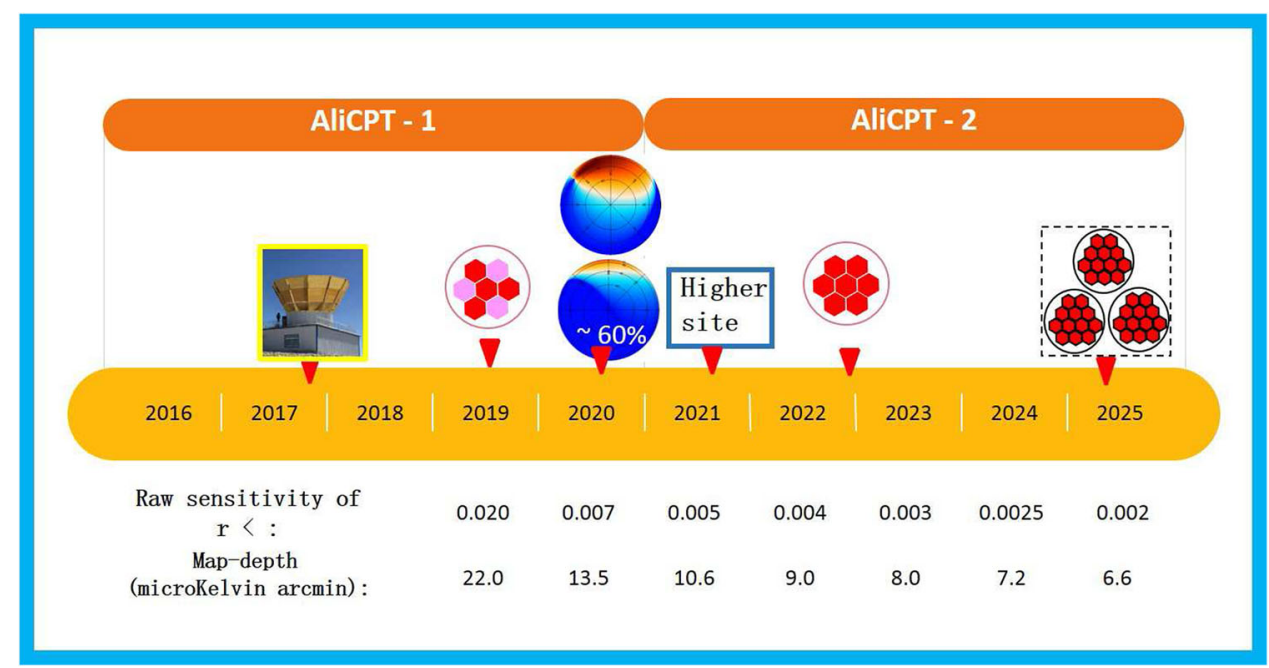


Acknowledgements This work is supported by Strategy Pilot B Programme of CAS (Grant No. XDB23020000), National Science Foundation of China (Grant Nos. 11653001,11653004), Key International S\&T Cooperation Projects of MOST (ministry of science and technology) (No. 2016YFE0104700).

Open Access This article is distributed under the terms of the Creative Commons Attribution 4.0 International License (http://creativecomm ons.org/licenses/by/4.0/), which permits unrestricted use, distribution, and reproduction in any medium, provided you give appropriate credit to the original author(s) and the source, provide a link to the Creative Commons license, and indicate if changes were made.

\section{References}

1. P.A.R. Ade et al., Keck array and BICEP2 collaborations, improved constraints on cosmology and foregrounds from BICEP2 and keck array cosmic microwave background data with inclusion of $95 \mathrm{GHz}$ band. Phys. Rev. Lett. 116, 031302 (2016)

2. J.A. Grayson, Ph.D. thesis, Stanford University, 2016

3. T. Matsumura et al., LiteBIRD: mission overview and focal plane layout. J. Low Temp. Phys. 184, 824-831 (2016)

4. J.Y. Suen et al., IEEE Trans. Terahertz Sci. Technol. 4(1), 86-100 (2014)

5. M.J. Griffin et al., The relative performance of filled and feedhorncoupled focal plane architectures. AO 41, 6543-6554 (2002)

6. J. Tolan, Ph.D. thesis, Stanford University, 2016

7. G.P. Teply, Ph.D. thesis, California Institute of Technology, 2015
8. D. Li et al., J. Low Temp. Phys. 184, 66-73 (2016)

9. C. Visnjic, Ph.D. thesis Princeton University, 2013

10. J.N. Ullom, D.A. Bennett, Review of superconducting transitionedge sensors for X-ray and gamma-ray spectroscopy. Supercond. Sci. Technol. 28, 084003 (2015)

11. C. Enss, Cryogenic Particle Detection Topics in Applied Physics, Chapter 1, vol. 99 (Springer, Berlin, 2005), pp. 1-34

12. K.D. Irwin, G.C. Hilton, Transition-Edge Sensors, Cryogenic Particle Detection, (Topics in Applied Physics), vol. 99 (Springer, Berlin, 2005), pp. 63-149

13. R.D. Planella, Ph.D. thesis, Pontificia Universidad Católica de Chile, 2009

14. M. Lueker, Ph.D. thesis, University of California, Berkeley, 2010

15. J.A. Brevik, Ph.D. thesis, California Institute of Technology, 2012

16. P.A.R. Ade et al., Astrophys. J. 812(176), 17 (2015)

17. J. Clarke, A.I. Braginski, The SQUID Handbook Volume I: Fundamentals and Technology of SQUIDs and SQUID Systems (Wiley Online Library, New Jersey, 2004)

18. D. Drung et al., Highly sensitive and easy-to-use SQUID sensors. IEEE Trans. Appl. Supercond. 17, 699-704 (2007)

19. P.A.J. de Korte et al., Time-division superconducting quantum interference device multiplexer for transition-edge sensors. Rev. Sci. Instrum. 74, 8 (2003)

20. M.P. Croce, et al., Preliminary assessment of microwave readout multiplexing factor, LA-UR-16-27204 (2017)

21. J. Beyer, D. Drung, A SQUID multiplexer with superconductingto-normal conducting switches. Supercond. Sci. Technol. 21, $105022(2008)$ 